\title{
Safety and efficacy of thromboprophylaxis with fondaparinux in elderly acutely ill medical patients with renal impairment: a retrospective single center study
}

\author{
Federico Silvestri, ${ }^{1}$ Samantha Pasca, ${ }^{2}$ Marco Zaramella, ${ }^{1}$ Antonella Labombarda, ${ }^{1}$ Andrea Barbi, ${ }^{1}$ Mauro Desideri, ${ }^{1}$ \\ Paolo Guidi, ${ }^{1}$ Adolfo Rogato, ${ }^{1}$ Matteo Bergamo, ${ }^{1}$ Giovanni Barillari ${ }^{2}$ \\ ${ }^{1}$ Division of Internal Medicine, General Hospital, Latisana (UD); ${ }^{2}$ Center for Hemorrhagic and Thrombotic Diseases, University \\ Hospital, Udine, Italy
}

\begin{abstract}
The majority of acutely ill medical patients are elderly with some degree of renal impairment. In this setting, venous thromboembolism (VTE) is one of the leading causes of morbidity and mortality and, to reduce this risk, a correct thromboprophylaxis is needed. The aim of this single center retrospective study was to assess the safety and efficacy of fondaparinux in elderly acutely ill medical patients with renal impairment. All patients aged 60 years or over, bedridden for at least four days, with a creatinine clearance $(\mathrm{CrCl})$ of $50 \mathrm{~mL} / \mathrm{min}$ or under, and who had received fondaparinux during hospitalization were evaluated and followed for up to 90 days after discharge. A total of 125 patients were evaluated ( $34.4 \%$ males); median age was 83.0 years. Median duration of thromboprophylaxis was 9.0 days. Forty-one (32.8\%) patients were treated with fondaparinux 1.5 $\mathrm{mg}$ daily, $84(67.2 \%)$ with $2.5 \mathrm{mg}$ daily. Inappropriately high doses of fondaparinux were used in 77 patients with $\mathrm{CrCl} 20-50$ $\mathrm{mL} / \mathrm{min}$, in 12 patients with $\mathrm{CrCl}$ below $20 \mathrm{~mL} / \mathrm{min}$, in 14 patients with prothrombin time (PT) ratio over 1.2, in 8 patients with PT ratio over 1.5, and in 3 patients with thrombocytopenia. No episodes of VTE or of major bleeding were recorded while there were 6 episodes $(2.4 \%)$ of minor bleeding. Both dosages of fondaparinux showed similar safety and efficacy. Twenty-six patients $(20.8 \%)$ died; no cause of death was related to fondaparinux. In conclusion, in elderly acutely ill hospitalized medical patients with renal impairment, prophylaxis with fondaparinux

Correspondence: Federico Silvestri, Division of Internal Med-

2.5 or $1.5 \mathrm{mg}$ daily is safe and effective in preventing VTE without increasing bleeding risk.
\end{abstract} icine, General Hospital, Latisana (UD), Italy.

Tel. +39.0431.529341.

E-mail: sampasca@alice.it; silvestrifederico@alice.it

Key words: fondaparinux, renal impairment, prophylaxis venous thromboembolism.

Acknowledgments: this is a retrospective, independent study, not supported by public or private companies.

Contributions: FS, SP and GB contributed equally to the study conception, interpretation of data and drafting the article; FS, $\mathrm{AL}, \mathrm{AB}, \mathrm{MD}, \mathrm{PG}, \mathrm{AR}, \mathrm{MZ}$ and $\mathrm{MB}$ contributed to data collection; FS, SP and GB contributed to interpretation and clinical revision of the manuscript. All authors approved the final version of the manuscript for publication. FS had full access to all the data and takes responsibility for its accuracy and analysis.

Conflict of interests: the authors declare no potential conflict of interests.

Received for publication: 14 April 2013.

Revision received: not required.

Accepted for publication: 2 May 2013.

This work is licensed under a Creative Commons Attribution NonCommercial 3.0 License (CC BY-NC 3.0).

CCopyright F. Silvestri et al., 2013

Licensee PAGEPress, Italy

Italian Journal of Medicine 2013; 7:287-292

doi:10.4081/itjm.2013.287

\section{Introduction}

Back in 1991, Anderson et al. ${ }^{1}$ demonstrated that the annual incidence of venous thromboembolism (VTE) among adults progressively increases with age, reaching $500-600$ cases per 100,000 of the population over the age of 80 years, which is the median age of the population of most divisions of Internal Medicine in Western countries.

Furthermore, VTE is one of the leading causes of morbidity and mortality in acutely ill medical patients. In fact, the incidence of deep vein thrombosis (DVT) and/or pulmonary embolism (PE) has been reported to range between $10 \%$ and $30 \%$ in hospitalized medical patients, a figure similar to that of surgical patients, ${ }^{2}$ and $\mathrm{PE}$ is thought to be associated with $5-10 \%$ of deaths of hospitalized patients, most of whom are medical patients. ${ }^{3}$ Large randomized controlled trials have demonstrated that prophylaxis with anticoagulant drugs reduces the risk of VTE, ${ }^{4-6}$ and in 2012 the American College of Chest Physicians (ACCP) recommended anticoagulant thromboprophylaxis with low molecular weight heparin (LMWH), low-dose unfractionated heparin, b.i.d., t.i.d., or fondaparinux (all Grade 1B) for high-risk hospitalized acutely ill medical patients. ${ }^{\text {? }}$ 
Several reports have shown that up to $40 \%$ of individuals hospitalized in Internal Medicine wards are carriers of some degree of renal impairment. ${ }^{8-9}$ As a consequence, they are at increased risk of both VTE and bleeding complications, ${ }^{10-13}$ particularly because they are older and have more co-morbidities than patients with normal renal function. Moreover, they frequently need thromboprophylaxis with anticoagulant drugs, such as LMWHs or fondaparinux, at predominant renal excretion and this is, therefore, potentially harmful since their bioaccumulation may result in excessive anticoagulant effect.

However, in general, there is little clinical information on safety and efficacy of fondaparinux in renal impaired patients and so far this been addressed in only one recent paper (the FONDAIR study). ${ }^{14}$

The aim of the present study was to evaluate safety and efficacy of fondaparinux in a 'real-life' context in a population of elderly hospitalized acutely ill medical patients with renal impairment.

\section{Materials and Methods}

We retrospectively reviewed the charts of all consecutive acutely ill medical patients over the age of 60 years admitted to the Internal Medicine Division of the Latisana Hospital, Italy, from January to June 2011 and given thromboprophylaxis with fondaparinux. Those with a creatinine clearance $(\mathrm{CrCl})$ below 50 $\mathrm{mL} / \mathrm{min}$ (part of a larger group of treated patients) were then evaluated for this retrospective single center study and make up this study cohort. Based on a local protocol, at hospital admission all patients received thromboprophylaxis if they were expected to remain in bed for at least four days and were affected by one of the following: congestive heart failure New York Heart Association (NYHA) class III or IV, acute respiratory failure, acute infection or inflammatory disorder, active cancer. Thromboprophylaxis was not administered if patients had active bleeding, bleeding events in the previous three months, known bleeding diathesis, ongoing oral anticoagulant treatment, known brain metastases, eye surgery, neurosurgery, spinal surgery, acute bacterial endocarditis or stroke within the previous 30 days.

At hospital admission, $\mathrm{CrCl}$ (calculated with the Cockroft-Gault formula ${ }^{15}$ ), platelet count (Plt) and prothrombin time (PT) ratio were evaluated before starting treatment.

According to the manufacturer's indications, fondaparinux (Arixtra ${ }^{\circledR}$, GlaxoSmithKline, Brentford, UK) $2.5 \mathrm{mg}$ s.c. daily should be given to patients with $\mathrm{CrCl}$ over $50 \mathrm{~mL} / \mathrm{min}$, Plt over $100 \times 10^{9} / \mathrm{L}$ and PT ratio below 1.2 ; and fondaparinux $1.5 \mathrm{mg}$ s.c. daily to patients with $\mathrm{CrCl} 20-50 \mathrm{~mL} / \mathrm{min}$, or Plt $50-100 \times 10^{9} / \mathrm{L}$ or PT ratio 1.2-1.5. Patients with $\mathrm{CrCl}$ below $20 \mathrm{~mL} / \mathrm{min}$, or Plt below $50 \times 10^{9} / \mathrm{L}$ or PT ratio over 1.5 should be excluded from thromboprophylactic treatment.

According to the local protocol, all patients were treated until hospital discharge, and they were subsequently followed-up after 90 days by means of a visit or a phone interview. Information on their health status, including the occurrence of symptomatic VTE or hemorrhagic events, was collected.

Major bleeding was defined according to the International Society of Thrombosis and Hemostasis guidelines: ${ }^{16}$ fatal bleeding, and/or symptomatic bleeding in a critical area/organ (intracranial, intraspinal, intraocular, retroperitoneal, intra-articular, pericardial, or intramuscular with compartment syndrome), and/or bleeding causing a fall in hemoglobin level of $2 \mathrm{~g} / \mathrm{dL}$ or more, or leading to transfusion of 2 or more units of whole blood or red cells. Clinically relevant nonmajor bleeding (CRNMB) was defined as any overt bleeding requiring medical intervention and/or treatment discontinuation and not meeting the criteria for major bleeding. Symptomatic DVT had to be confirmed by Doppler ultrasound; symptomatic PE by helical computed tomography. Both bleeding events and DVT episodes were respectively considered as side effects or lack of efficacy of fondaparinux if they occurred during administration of the drug or in the $48 \mathrm{~h}$ following suspension.

\section{Statistical analysis}

Continuous variables were expressed as a mean plus or minus the standard deviation (SD) or as a median with range. Univariate analysis of base-line characteristics and prevalence of potential risk factors in patients with $\mathrm{CrCl}$ over or below $50 \mathrm{~mL} / \mathrm{min}$ was performed by t-test in case of continuous variables, and by $\chi^{2}$ test or Fisher's exact test in case of categorical variables. Data were analyzed using $\mathrm{R}$ (version 2.15.0) software. $\mathrm{P}<0.05$ was considered significant.

\section{Results}

\section{Patients}

Over the 6-month study period, 210 consecutive patients admitted to the Internal Medicine division who met inclusion criteria for thromboprophylaxis, received treatment with fondaparinux. For the purpose of this study, only the 125 patients with a $\mathrm{CrCl}$ below $50 \mathrm{~mL} / \mathrm{min}$ are reported. Base-line characteristics of this population are summarized in Table 1.

Briefly, 43 (34.4\%) patients were male, mean age was $83.2 \pm 7.3$ years and median age 83.0 (64-98) years. Creatinine clearance was $20-50 \mathrm{~mL} / \mathrm{min}$ in 113 (90.4\%) patients and below $20 \mathrm{~mL} / \mathrm{min}$ in 12 patients $(9.6 \%)$. Table 1 also shows the main diseases for 
which patients were admitted to the hospital and given thromboprophylaxis, and main concomitant disorders.

Of 125 patients, 77 (61.6\%) were receiving concomitant anti-platelet agents; the majority of these patients $(n=65,84.4 \%)$ were on aspirin, 7 were receiving ticlopidine (9.1\%) and 5 were receiving dual antiplatelet therapy with aspirin and clopidogrel (6.5\%).

Overall, fondaparinux was used at the dose of 1.5 $\mathrm{mg}$ daily in $41(32.8 \%)$ patients, and at $2.5 \mathrm{mg}$ daily in $84(67.2 \%)$.
Mean duration of hospitalization was $13.7 \pm 10.9$ days and mean duration of thromboprophylaxis with fondaparinux was 12.3 \pm 11.9 days (range 5-69). In particular, fondaparinux was administered for up to 14 days, according to current guidelines, in 96 patients $(75.6 \%)$ and for over 14 days in the remaining patients.

Table 2 shows the relationship between $\mathrm{CrCl}$, PT ratio, platelet count and the actual dose of fondaparinux used: a high dose of fondaparinux $(2.5 \mathrm{mg}$

Table 1. Patients' characteristics.

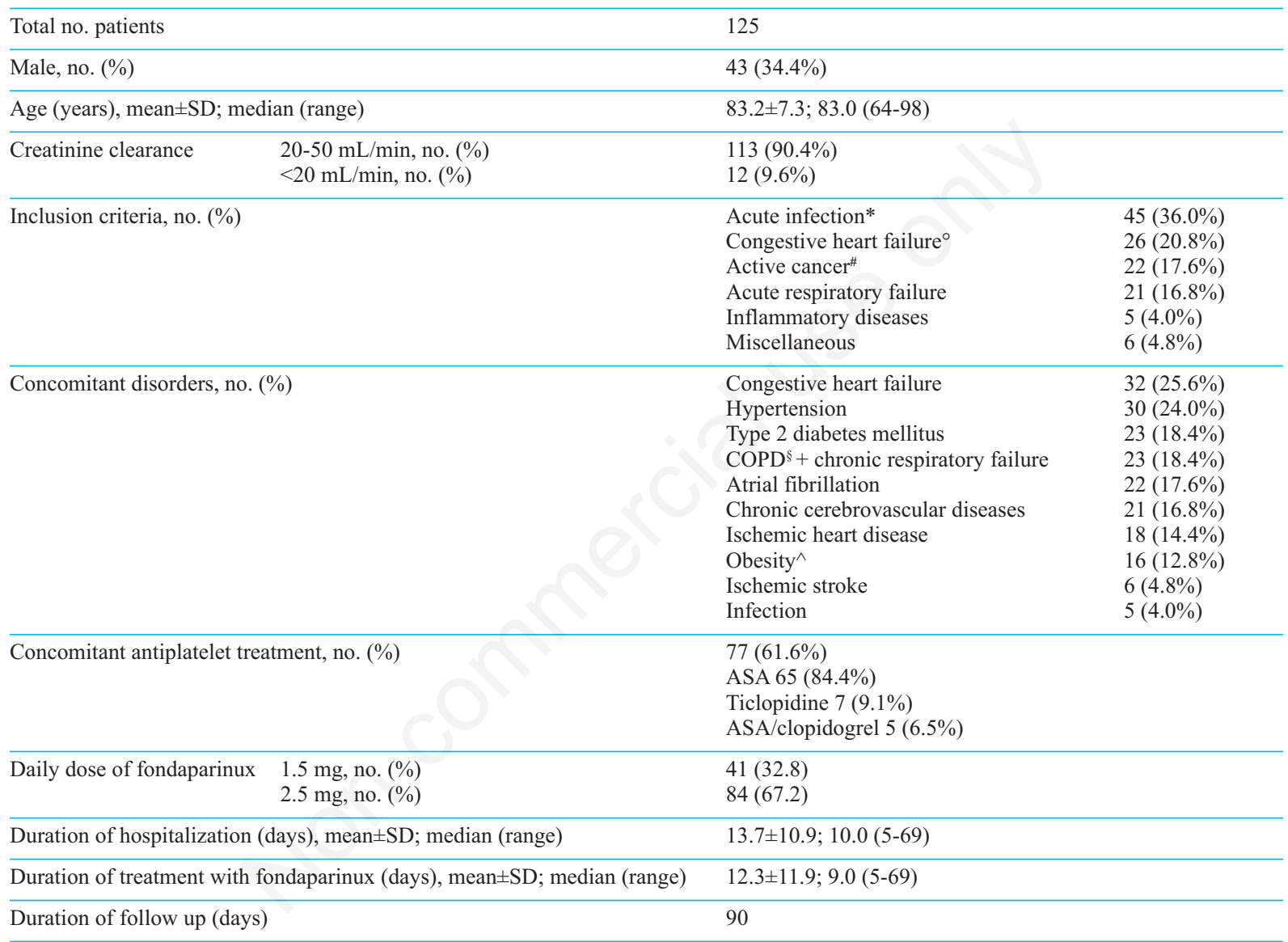

*Including lung infections (pneumonia, bronchitis and exacerbated chronic obstructive pulmonary disease) and other infections (i.e. sepsis, cholecystitis, urinary tract infection and erysipelas); ${ }^{\circ} \mathrm{NYHA}$ class III-IV; ${ }^{*}$ on chemotherapy or in progression on supportive care; ${ }^{\S}$ chronic obstructive pulmonary disease; ${ }^{\wedge} \mathrm{BMI} \geq 30$ for males and $\geq 28.6$ for females.

Table 2. Relationship between creatinine clearance, prothrombin time ratio, platelet count and fondaparinux dose used.

\begin{tabular}{|c|c|c|c|c|}
\hline & & $\begin{array}{l}\text { All patients } \\
\text { (no. 125) }\end{array}$ & $\begin{array}{c}\text { Fondaparinux } \\
1.5 \mathrm{mg} / \text { die (no. 41) }\end{array}$ & $\begin{array}{c}\text { Fondaparinux } \\
2.5 \mathrm{mg} / \mathrm{die}(\text { no. 84) }\end{array}$ \\
\hline $\mathrm{CrCl} \mathrm{mL} / \mathrm{min}$ & $\begin{array}{l}20-50 \\
<20\end{array}$ & $\begin{array}{c}113(90.4 \%) \\
12(9.6 \%)\end{array}$ & $\begin{array}{c}36(87.8 \%) \\
5(12.2 \%)\end{array}$ & $\begin{array}{c}77(91.7 \%) \\
7(8.3 \%)\end{array}$ \\
\hline PT ratio & $\begin{array}{c}<1.2 \\
1.2-1.5 \\
>1.5\end{array}$ & $\begin{array}{c}90(72.0 \%) \\
27(21.6 \%) \\
8(6.4 \%)\end{array}$ & $\begin{array}{c}24(58.5 \%) \\
13(31.7 \%) \\
4(9.7 \%)\end{array}$ & $\begin{array}{c}66(78.6 \%) \\
14(16.7 \%) \\
4(4.7 \%)\end{array}$ \\
\hline Platelets $\times 10^{9} / \mathrm{L}$ & $\begin{array}{l}\geq 100 \\
<100 \\
<50\end{array}$ & $\begin{array}{c}121(96.8 \%) \\
3(2.4 \%) \\
1(0.8 \%)\end{array}$ & $\begin{array}{c}40(97.6 \%) \\
1(2.4 \%) \\
0\end{array}$ & $\begin{array}{c}81(96.4 \%) \\
2(2.4 \%) \\
1(1.2 \%)\end{array}$ \\
\hline
\end{tabular}

$\mathrm{CrCl}$, creatinine clearance; PT, prothrombin time. 
daily) was inappropriately used in 84 patients with $\mathrm{CrCl}$ below $50 \mathrm{~mL} / \mathrm{min}$ (7 of whom with $\mathrm{CrCl}<20$ $\mathrm{mL} / \mathrm{min}$ ). Of these, 18 patients showed also a PT ratio over 1.2 and 3 patients a Plt count below $100 \times 10^{9} / \mathrm{L}$; no bleeding was observed in this group of patients. Fondaparinux at $1.5 \mathrm{mg}$ daily was also inappropriately used in 5 patients with $\mathrm{CrCl}$ below $20 \mathrm{~mL} / \mathrm{min}$ (4 of whom showed also a PT ratio $>1.2$ ) and in 4 patients with a PT ratio over 1.5; again no bleeding was observed also in this group of patients.

\section{Safety}

Study outcomes are described in Table 3. No major bleedings were observed.

Three CRNMB episodes $(2.4 \%)$ were registered: one (melena) in a patient with lung cancer and liver cirrhosis receiving fondaparinux $1.5 \mathrm{mg}$ daily plus ASA and a PT ratio of 1.27; one (melena) in an 86year old female with diabetes mellitus and chronic cerebrovascular disease, receiving fondaparinux 1.5 $\mathrm{mg}$ daily; and one (epistaxis and gum bleeding) in a patient with pharyngeal cancer programmed for chemo- and radiotherapy, receiving fondaparinux 1.5 $\mathrm{mg}$ daily. In all these cases, fondaparinux was continued. Another episode of CRNMB (hematuria) occurred after discharge in a patient receiving aspirin. The event occurred 17 days after fondaparinux had been stopped and is, therefore, not counted as being related to thromboprophylaxis.

When comparing the base-line characteristics of patients with $\mathrm{CrCl}$ below or above $50 \mathrm{~mL} / \mathrm{min}$ at univariate analysis (Table 4), bleeding events were equally distributed among the two groups, even though the group with renal impairment was significantly older, and with more patients aged over 80 years and on antiplatelet therapy.

\section{Efficacy}

While no DVT episodes were registered during administration of fondaparinux, only one case was registered during follow up (upper arm, catheter-related), but this must not be included in the study outcomes since it occurred at Day 67 after discharge and discontinuation of fondaparinux in a patient with urothelial cancer of bladder.

\section{Mortality}

During the observation period, 26 of 125 patients (20.8\%) died. Only 5 (19.2\%) of these died during hospitalization while on thromboprophylaxis ( 2 multiorgan failures, 2 cancers and one infection); the others died either during follow up or off therapy. Causes of death are summarized in Table 5; no cause of death was related to fondaparinux.

Table 3. Study outcomes.

\begin{tabular}{lcc}
\hline Outcome & No. events & \% \\
\hline Major bleeding & 0 & - \\
\hline CRNMB & 3 & 2.4 \\
\hline Symptomatic VTE & 0 & - \\
\hline
\end{tabular}

CRNMB, clinically relevant non-major bleeding; VTE, venous thromboembolism.

Table 4. Comparison of characteristics of patients with creatinine clearance $<$ or $\geq 50 \mathrm{~mL} / \mathrm{min}$.

\begin{tabular}{|c|c|c|c|}
\hline Characteristics & $\begin{array}{c}\mathrm{CrCl}<50 \mathrm{~mL} / \mathrm{min} \\
\text { no. } 125\end{array}$ & $\begin{array}{c}\mathrm{CrCl} \geq 50 \mathrm{~mL} / \mathrm{min} \\
\text { no. } 85\end{array}$ & $\mathbf{P}$ \\
\hline Male, no. $(\%)$ & $43(34.4 \%)$ & $48(56.5 \%)$ & $<0.05$ \\
\hline Age (years), mean $\pm \mathrm{SD}$ (range) & $83.2 \pm 7.3(64-98)$ & $75.1 \pm 7.7(64-88)$ & $<0.05$ \\
\hline Age $\geq 80$ years, no. $(\%)$ & $86(68.8)$ & $27(32.1)$ & $<0.05$ \\
\hline Bleeding events, no. (\%) & $3(2.4 \%)$ & $4(4.7 \%)$ & 0.352 \\
\hline Antiplatelet therapy, no. (\%) & $77(61.6 \%)$ & $40(47.0 \%)$ & $<0.05$ \\
\hline Fondaparinux dose 2.5 , no. (\%) & $84(67.2)$ & $56(65.9)$ & 0.936 \\
\hline $\mathrm{PT}$ ratio $>1.2$, no. $(\%)$ & $34(27.2)$ & $21(24.7)$ & 0.698 \\
\hline Platelets $<100 \times 10^{9} / \mathrm{L}$, no. $(\%)$ & $4(3.2)$ & $6(7.1)$ & 0.190 \\
\hline Active cancer, no. $(\%)$ & $22(17.6)$ & $17(20.0)$ & 0.631 \\
\hline Obesity & $16(12.8)$ & $29(34.1)$ & $<0.05$ \\
\hline Symptomatic VTE & 0 & $1(1.2)$ & 0.405 \\
\hline
\end{tabular}

$\mathrm{CrCl}$, creatinine clearance; PT, prothrombin time; VTE, venous thromboembolism. 
Table 5. Causes of death.

\begin{tabular}{lc}
\hline Causes of death & No. patients $(\%)$ \\
\hline Died & $26(20.8 \%)$ \\
\hline Cancer & $9(34.6 \%)^{*}$ \\
\hline Multiorgan failure & $8(30.8 \%)$ \\
\hline Chronic heart failure & $5(19.2 \%)$ \\
\hline Infection & $2(7.7 \%)$ \\
\hline Complications of AMI & $1(3.8 \%)$ \\
\hline Unknown & $1(3.8 \%)$
\end{tabular}

*Percentage of total number of patients who died $(n=26)$. AMI, acute myocardial infarction.

\section{Discussion}

As compared to the usual $2.5 \mathrm{mg}$ daily-dose of fondaparinux, a lower dose (1.5 mg daily) was recently approved for the prevention of VTE in patients with renal impairment, after data had been obtained in patients undergoing major orthopedic surgery. ${ }^{17}$ This lower dose has been prospectively tested in only one recently published multicenter study on patients affected by renal impairment (creatinine clearance between 20 and 50 $\mathrm{mL} / \mathrm{min}$ ) (the FONDAIR study). ${ }^{14}$ The characteristics of the patient population in this study were similar to those in ours. In fact, these included 206 patients with a mean age of 82.1 years, with multiple co-morbidities, with a high percentage of patients being affected by cancer $(21.8 \%)$ and receiving antiplatelet medication $(62.1 \%)$. The observed incidence of major bleeding $(0.49 \%)$, of CRNMB $(3.88 \%)$, and of symptomatic VTE $(1.46 \%)$ prompted the authors of the study to indicate fondaparinux at $1.5 \mathrm{mg}$ daily as safe and effective for the prevention of VTE in acutely ill medical patients with renal impairment.

In our study, the dose of fondaparinux should have been used according to the manufacturer's instructions (after evaluating creatinine clearance, Plt count and PT ratio). However, when retrospectively analyzing hospital charts, we realized that many daily doses administered to patients were higher ( $2.5 \mathrm{mg}$ daily) than those advised. Nevertheless, when comparing our safety data with those of the FONDAIR study and of the three large randomized studies on VTE prophylaxis with fondaparinux, enoxaparin and deltaparin, ${ }^{4-6}$ we noted that, in our population, the incidence of bleeding episodes (major and non-major) was even lower than that reported by those trials, even among patients in whom non-appropriate doses of fondaparinux were used, i.e. $2.5 \mathrm{mg}$ daily in patients with a $\mathrm{CrCl} 20-50 \mathrm{~mL} / \mathrm{min}$ or PT ratio over 1.2 or Plt below $100 \times 10^{9} / \mathrm{L}$ or $1.5 \mathrm{mg}$ daily in patients with a $\mathrm{CrCl}$ below $20 \mathrm{~mL} / \mathrm{min}$ or PT ratio over 1.5 .

These safety issues appear to be of particular im- portance considering the population studied, i.e. a very old population, older than that of the three randomized studies mentioned above ( 83.0 vs 75.0, 73.1 and 68.5, respectively), with a greater percentage of patients affected by cancer (17.6 vs 14.5, 12.3 and 4.6, respectively) and a higher number of deaths (20.8 vs 3.3, 11.4 and 6.1 , respectively). In other words, a population with no base-line selection of patients and poorly represented in those randomized studies, i.e. the reallife elderly acutely ill patients admitted onto medical wards. This is also confirmed by the unsurprisingly high fatality rate reported in our study (20.8\%), as compared to the $4.3 \%$ of a meta-analysis of randomized studies in patients receiving active treatment ${ }^{18}$ and to the $4.9 \%$ of the Lifenox study, ${ }^{19}$ given the mean age of the population and, most of all, the severity of comorbidities (Table 1).

As far as efficacy is concerned, no VTE episodes were observed in our study among patients receiving thromboprophylaxis, while the incidence of symptomatic VTE was higher (0.38\% DVT and $0.20 \%$ PE) in the meta-analysis of Dentali, ${ }^{18}$ and even higher again $(1.46 \%)$ in the FONDAIR study. ${ }^{14}$ In their conclusions, the authors of this last study questioned whether a higher dose of fondaparinux (i.e. $2.5 \mathrm{mg}$ ) would have been more effective and whether it would have been able to reduce this rate. Our data on the safety of the $2.5 \mathrm{mg}$ daily dose in a population with renal impairment might in some way answer this question, suggesting the need for a direct comparison between the higher and the lower dose of the drug to provide concrete evidence as to which approach is associated with the most favorable clinical benefit in this population.

Our study has a number of limitations: i) its retrospective nature; ii) it was carried out in a single center; iii) only a small number of patients were enrolled; iv) asymptomatic deep vein thrombosis was not routinely screened and some events could have been missed; and v) the method used to estimate renal function might not have been the most appro- 
priate. Nevertheless, it opens up some interesting points for debate since it shows that the use of fondaparinux is a safe and effective strategy to prevent VTE in elderly acutely ill medical patients with renal impairment, with a very low incidence of bleeding complications.

\section{References}

1. Anderson FA, Wheeler HB, Goldberg RJ, et al. A population-based perspective of the hospital incidence and case-fatality rates of deep vein thrombosis and pulmonary embolism. The Worcester DVT study. Arch Intern Med 1991;151:933-8.

2. Geerts WH, Pineo GF, Heit JA, et al. Prevention of venous thromboembolism; the Seventh ACCP Conference on Antithrombotic and Thrombolytic Therapy. Chest 2004; 126:338S-400S.

3. Cohen AT, Edmonson RA, Phillips MJ, et al. The changing pattern of venous thromboembolic disease. Haemostasis 1996;26:65-71.

4. Cohen AT, Davidson BL, Gallus AS, et al. Efficacy and safety of fondaparinux for the prevention of venous thromboembolism in older acute medical patients: randomised placebo controlled trial. BMJ 2006;332:325-9.

5. Samana MM, Cohen AT, Darmon JY, et al. A comparison of Enoxaparin with placebo for the prevention of venous thromboembolism in acutely ill medical patients. NEJM 1999;341:793-800.

6. Leizorovicz A, Cohen AT, Turpie AGG, et al. Randomized, placebo-controlled trial of Deltaparin for the prevention of venous thromboembolism in acutely ill medical patients. Circulation 2004;110:874-9.

7. Guyatt GH, Akl EA, Crowther M, et al. Antithrombotic therapy and prevention of thrombosis. 9 th ed., American College of Chest Physicians. Evidence based clinical practice guidance. Executive summary. Chest 2012;141:7S-47S.

8. Gussoni G, Campanini M, Silingardi M, et al. In hospital symptomatic venous thromboembolism and antithrombotic prophylaxis in internal medicine. Findings from a multicenter, prospective study. Throm Haemost 2009;101:893-901.
9. Dentali F, Riva N, Gianni M, et al. Prevalence of renal failure and use of thromboembolic prophylaxis among medical inpatients at increased risk of venous thromboembolic events. Thromb Res 2008;123:67-71.

10. Monreal M, Falgà C, Valle R, et al. Venous thromboembolism in patients with renal insufficiency: findings from the RIETE registry. Am J Med 2006;119:1073-9.

11. Wattanakit K, Cushman M, Stehman-Breen C, et al. Chronic kidney disease increases risk for venous thromboembolism. J Am Soc Nephrol 2008;19:135-40.

12. Parikh AM, Spencer FA, Lessard D, et al. Venous thromboembolism in patients with reduced estimated GFR: a population based prospective. Am J Kidney Dis 2011; 58:746-55.

13. Decousous H, Tapson VF, Bergmann JF, et al. Factors at admission associated with bleeding risk in medical patients: findings from the IMPROVE investigators. Chest 2011;139:69-79.

14. Ageno W, Riva N, Noris P, et al. Safety and efficacy of low dose fondaparinux $(1.5 \mathrm{mg})$ for the prevention of venous thromboembolism in acutely ill medical patients with renal impairment. The FONDAIR study. J Thromb Haemost 2012;10:2291-7.

15. Cockroft DW, Gault MH. Prediction of creatinine clearance from serum creatinine. Nephron 1976;16:31-41.

16. Schulman S, Keraon C; the subcommittee on control of anticoagulation of the scientific and standardization committee of ISTH. Definition of major bleeding in clinical investigations of antihemostatic medicinal products in non-surgical patients. J Thromb Haemost 2005; 3:692-4.

17. Turpi AGG, Lensing AWA, Fuji T, Boyle DA. Pharmacokinetic and clinical data supporting the use of fondaparinux $1.5 \mathrm{mg}$ once daily in the prevention of venous thromboembolism in renally impaired patients. Blood Coagul Fibrinolysis 2009;20:114-21.

18. Dentali F, Douketis JD, Gianni M, et al. Metaanalysis: anticoagulant prophylaxis to prevent symptomatic venous thromboembolism in hospitalized medical patients. Ann Inter Med 2007;146:278-88.

19. Kakkar AK, Cimminiello C, Goldhaber SZ, et al. Low molecular weight heparin and mortality in acutely ill medical patients. N Engl J Med 2011;365:2463-72. 